\title{
Location of the North Magnetic Pole in April 2007
}

\author{
L. R. Newitt ${ }^{1}$, A. Chulliat ${ }^{2}$, and J.-J. Orgeval ${ }^{3}$ \\ 1310-1171 Ambleside Drive, Ottawa, Canada K2B 8E1 \\ ${ }^{2}$ Equipe de Géomagnétisme, Institut de Physique du Globe de Paris, 4 Place Jussieu, 75005 Paris, France \\ ${ }^{3}$ 16, Allé du Houx 45160 Olivet, France
}

(Received January 8, 2008; Revised November 20, 2008; Accepted November 22, 2008; Online published July 27, 2009)

\begin{abstract}
Observations have been made at five locations in the vicinity of the North Magnetic Pole (NMP). These were used in four different analyses_-virtual geomagnetic pole, simple polynomial, spherical cap harmonic, best fitting grid - to derive positions of the NMP. The average position at 2007.3 was $83.95^{\circ} \mathrm{N}, 120.72^{\circ} \mathrm{W}$, with a positional uncertainty of $40 \mathrm{~km}$. This position is only $27 \mathrm{~km}$ from the pole position given by the CHAOS magnetic model. The NMP continues to move in a northwesterly direction but its drift speed has stabilized at just over $50 \mathrm{~km}$ per year. The number of direct observations is insufficient to determine if the NMP has started to decelerate.
\end{abstract}

Key words: North Magnetic Pole, secular variation, geomagnetic models, geomagnetic jerks.

\section{Introduction}

The North Magnetic Pole (NMP) is a geophysical phenomenon that is often misunderstood. Common but erroneous beliefs include the following: The NMP attracts compass needles; compasses point directly at the NMP; a compass needle spins uncontrollably on its pivot at the NMP; the field is strongest at the pole; the magnetic field at the NMP in some way affects health; compasses point to the South Magnetic Pole in the southern hemisphere. The release of a new position for the NMP presents an ideal opportunity for public education aimed at correcting some of these misconceptions.

The NMP is defined as the point on the Earth's surface at which the Earth's magnetic field is directed vertically downward. At the NMP, inclination is $90^{\circ}$ and horizontal intensity is zero. Although all lines of equal magnetic declination converge on the NMP, declination at the NMP itself is undefined. Magnetic meridians also converge on the NMP, but, as a result of the extreme non-dipole nature of the magnetic field in the north polar region, this convergence is not radial. Thus, although all compasses lead to the NMP, they do not point directly at it.

The magnetic poles must be distinguished from other members of the "Pole" family such as the "geomagnetic pole", the "eccentric pole", the "geographic/rotational pole", the "pole of inaccessibility" to name a few. A good discussion of the geomagnetic and eccentric poles is given by Fraser-Smith (1987). Lowes (1994) also gives a detailed description of the eccentric pole. Table 1 summarizes the definitions of the (geo)magnetic poles.

Implicit in the definition of the NMP is the assumption that the magnetic field is entirely of internal (core) origin, and that its movement is due to the geomagnetic secular

Copyright (c) The Society of Geomagnetism and Earth, Planetary and Space Sciences (SGEPSS); The Seismological Society of Japan; The Volcanological Society of Japan; The Geodetic Society of Japan; The Japanese Society for Planetary Sciences; TERRAPUB. variation originating with fluid motions in the core. In practice, it is impossible to observe the core field alone since external fields and lithospheric fields are always present. The observer can only try to reduce the errors caused by these fields by not observing when the field is active and by not observing in the vicinity of known crustal anomalies. Campbell (2003) has stated that these two factors render suspect any magnetic pole position obtained through local surveys; in his opinion, the positions of the magnetic poles can only be obtained from spherical harmonic models. We agree in part with Campbell. The large volume of satellite data that has been gathered since the launch of Ørsted and the thoroughness of the processing should result in better separation or core, crust and external fields than is possible with an analysis of only a few surface observations. However, the field due to the core and the field due to long wave-length crustal anomalies overlap. If one excludes these lithospheric sources from a model by choosing a low degree and order spherical harmonic expansion, one will also exclude part of the core field.

Magnetic cartography was the initial reason for determining the NMP location. James Ross, the first person to locate the NMP, wrote "the determination of the position of the Magnetic Poles of the earth has ever been considered a desideratum in the science of magnetism of the highest importance." (Ross, 1834). As the importance of charts waned in favour of reference field models, determining the pole position became a way of validating the model in a region of the Earth where good quality data were sparse. However, the high quality of recent reference field models puts into question the continued need for direct observation of the NMP.

For much of the twentieth century the NMP maintained a northward migration of about $10 \mathrm{~km}$ per year. Sometime during the last couple of decades of that century, something triggered a remarkable acceleration in its movement. This was first noticed by Newitt and Barton (1996) after a sur- 
Table 1.

\begin{tabular}{ll}
\hline \multicolumn{1}{c}{ Pole } & \multicolumn{1}{c}{ Definition } \\
\hline \hline $\begin{array}{l}\text { Geomagnetic } \\
\text { (Centred dipole) }\end{array}$ & $\begin{array}{l}\text { the two points on the Earth's surface formed by the axis of an inclined dipole placed } \\
\text { at the centre of the Earth; position calculated from the first three coefficients of } \\
\text { a spherical harmonic model }\end{array}$ \\
\hline \multirow{3}{*}{$\begin{array}{l}\text { the two points on the Earth's surface formed by the axis of an inclined dipole displaced } \\
\text { from the centre of the Earth; calculated from spherical harmonic coefficients using } \\
\text { the same orientation and moment as the centred dipole }\end{array}$} \\
$\begin{array}{l}\text { Magnetic } \\
\text { (dip) }\end{array}$ \\
$\begin{array}{l}\text { the two points on the Earth's surface at which the Earth's magnetic field is directed vertically } \\
\text { procedure or from a local magnetic survey; the only pole that is directly observable }\end{array}$
\end{tabular}

vey carried out in 1994. (Although the acceleration of the NMP could have been detected using positions calculated from reference field models, it was the act of verifying the position derived from the observations that drew their attention to the acceleration.) Newitt et al. (2002), using data from a survey carried out in 2001, confirmed that the rapid acceleration had continued, and that the rate of motion in 2001 was about $40 \mathrm{~km}$ per year. This differs considerably from the speed of the South Magnetic Pole, which is about $4 \mathrm{~km}$ per year (Mandea and Dormy, 2003). Newitt et al. (2002) also speculated that the increase in the speed of the NMP could be due to magnetic jerks. Mandea and Dormy (2003) show that a strong correlation exists between jerks and changes in the NMP velocity.

In this paper, we derive a location for the NMP based primarily on observations made in April, 2007 during the latest Polyarctic expedition. We compare this and previous positions with positions derived from various spherical harmonic models such as the IGRF. Finally, we discuss the need for, and the practicality of continuing, NMP surveys. In a companion paper, we will discuss the field at the core mantle boundary and how this relates to the movement of the NMP.

\section{The 2007 Survey-Observations and Data Re- duction}

\subsection{Survey logistics}

A generic North Magnetic Pole survey consists of taking observations of declination $(D)$, inclination $(I)$ and total intensity $(F)$ at several spots around the location where the NMP is expected to be found based on an extrapolation of past positions. The sites are accessed by fixed-winged aircraft, specifically a De Havilland DHC Twin Otter equipped with skis. The survey is carried out in late April or early May, a period of continuous sunlight, bearable temperatures $\left(-20^{\circ} \mathrm{C}\right)$ and relatively stable ice. Once on the ice, the observing procedure is similar to that carried out at a magnetic repeat station (see Newitt et al., 1996). However, we allow only one hour to set up and observe, compared to up to three days at a repeat station. Declination and inclination are measured using a declination-inclination magnetometer, consisting of a fluxgate sensor mounted on a steel-free theodolite. The theodolite is also used to sight the sun for the purpose of determining the true north direction. Position is obtained from the aircraft's GPS. Total intensity is measured using an Overhauser magnetometer from Gem Systems Inc. Logistical considerations determine whether a fluxgate variometer is installed in the survey area.

Prior to the 2007 survey, we estimated that the NMP would be near $84^{\circ} \mathrm{N}, 124^{\circ} \mathrm{W}$, more than $710 \mathrm{~km}$ northwest Eureka Weather Station, the nearest inhabited location. This placed the NMP beyond the range of the Twin Otter, which meant arranging for a second aircraft to bring in fuel. The distance between the NMP and the base of operations also consumed approximately 6 hours of flying time, thereby limiting to four the number of observation sites that could be visited in the single day during which the aircraft was available to us. These factors also precluded the installation of a variometer.

\subsection{Observations}

Figure 1 shows the location of the four observation points of the 2007 survey. A similar survey had been attempted in 2005, but was cut short because of bad weather after only one usable observation had been made. This observation is also shown on the map. The observed values are given in Table 2. For Site 05-01, both observed (bracketed) and updated to 2007.3 (unbracketed) values are given.

The observations contain errors from a number of sources: incomplete demagnetization of the observer, observational errors in both the sun shots and the $D$ and $I$ readings, and the effects of external magnetic fields. External fields constitute the source of the largest error.

Ideally, one should correct the observations to the quiet level of the magnetic field, but determining the quiet level of the field is extremely difficult during the summer months in the polar cap, even at a magnetic observatory. In practice, the problem is made worse by the great distance between the NMP and the nearest magnetic observatory. Resolute Bay is approximately $1160 \mathrm{~km}$ distant; the magnetometer installation at Eureka (not a full observatory) is $710 \mathrm{~km}$ distant. Figures 2 and 3 show plots of declination and inclination on April 29 and 30 at both Eureka and Resolute. It can be seen that although there are some similarities between the variations in $D$ and $I$ at Eureka and Resolute, differences can be large. In fact, differences between the two curves are approximately the same magnitude as the daily variation. Although we corrected each componentobservation to the estimated average nighttime value at the time of the survey, the resultant values still contain considerable error and are probably not much better than the uncorrected values.

In order to estimate the error in the observations, we examined hourly mean values at Resolute Bay observatory. Thirty-two days, with activity levels comparable to the days 


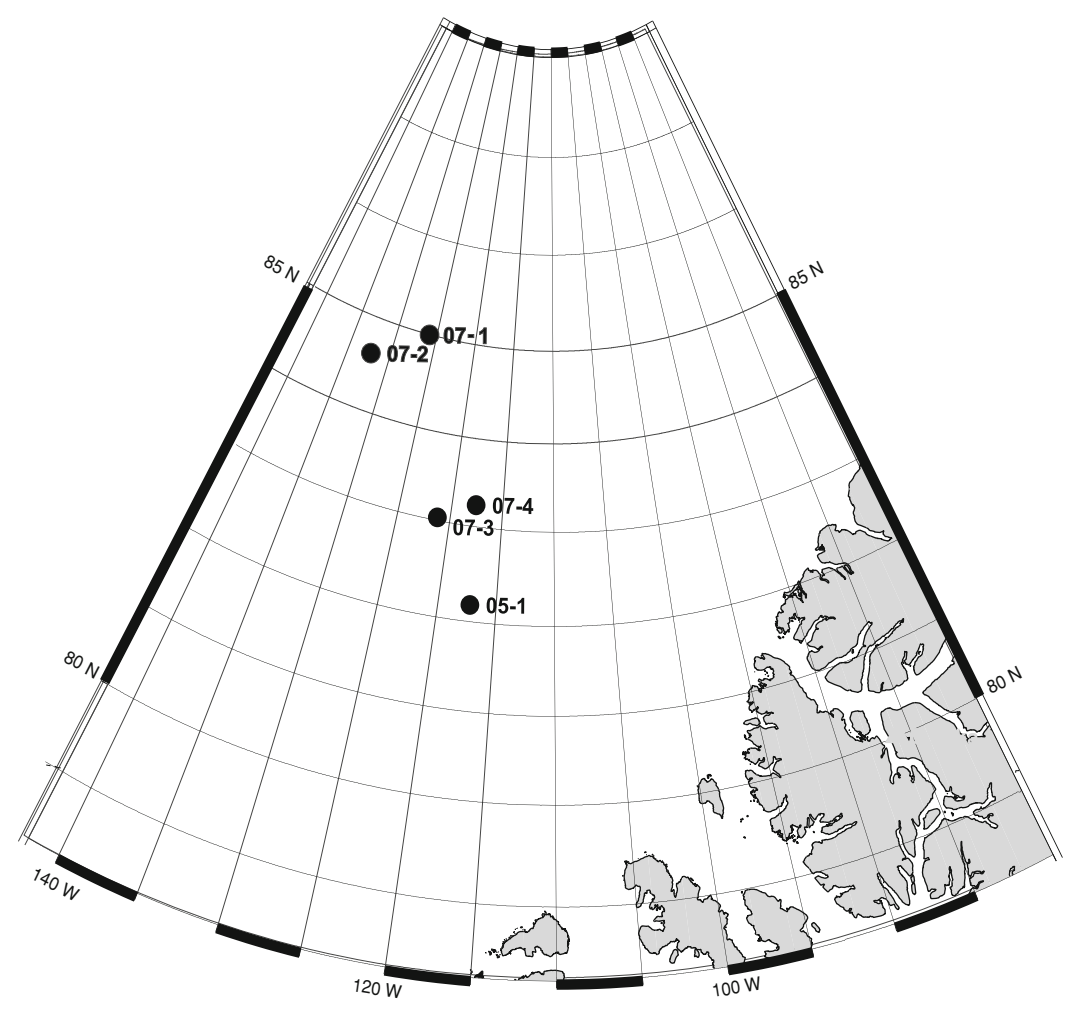

Fig. 1. Location of observations used to determine the position of the North Magnetic Pole.

Table 2.

\begin{tabular}{llllll}
\hline & \multicolumn{1}{c}{ Site $07-1$} & \multicolumn{1}{c}{ Site $07-2$} & \multicolumn{1}{c}{ Site $07-3$} & \multicolumn{1}{c}{ Site $07-4$} & \multicolumn{1}{c}{$\begin{array}{c}\text { Site } 05-1 \text { updated } \\
\text { (observed) }\end{array}$} \\
\hline Date & $2007-04-29$ & $2007-04-29$ & $2007-04-29$ & $2007-04-30$ & $2005-05-18$ \\
Time & $18: 03$ to $18: 22$ & $19: 40$ to $20: 12$ & $22: 15$ to $22: 35$ & $00: 18$ to $00: 46$ & \\
Latitude (N) & $84^{\circ} 59.505$ & $84^{\circ} 36.546^{\prime}$ & $83^{\circ} 04.912$ & $83^{\circ} 16.576$ & $82^{\circ} 10.9^{\prime}$ \\
Longitude (W) & $125^{\circ} 57.20$ & $132^{\circ} 16.888^{\prime}$ & $121^{\circ} 10.340^{\prime}$ & $117^{\circ} 38.0348$ & $117^{\circ} 14.8$ \\
$D$ & $-120^{\circ} 08.4$ & $62^{\circ} 22.7^{\prime}$ & $91^{\circ} 19.3^{\prime}$ & $-143^{\circ} 06.6^{\prime}$ & $18^{\circ} 45^{\prime} \mathrm{E}\left(25^{\circ} 18^{\prime} \mathrm{E}\right)$ \\
$I$ & $89^{\circ} 50.6$ & $89^{\circ} 24.8$ & $89^{\circ} 50.6$ & $89^{\circ} 47.7$ & $89^{\circ} 06\left(89^{\circ} 09^{\prime}\right)$ \\
$F$ & 56849 & 57125 & 56994 & 57210 & $57195(57145)$ \\
$X$ & -78 & 271 & -4 & -164 & $854(766)$ \\
$Y$ & -134 & 532 & 156 & -123 & $290(362)$ \\
$Z$ & 56648 & 57122 & 56993 & 57209 & $57188(57138)$ \\
\hline
\end{tabular}

on which we observed, were selected from the months of April and May. Differences between the hourly means and the mean value for the two month period were calculated. We found that $95 \%$ of the hourly declination values were within $2.8^{\circ}$ of the mean declination and $95 \%$ of the inclination observations fell within the 0.1 degrees of the mean inclination. This must be considered a best case scenario since we did not observe over a full hour and the variation over an hour can easily exceed $100 \mathrm{nT}$, which is comparable to the variation between hourly means. Combining variances gives an estimated error of $4.0^{\circ}$ for declination and $0.14^{\circ}$ for inclination. The error in declination is highly dependent on the strength of the horizontal field; an error of $4.0^{\circ}$ at Resolute scales to errors that range from $20^{\circ}$ to $40^{\circ}$ at the observation points. However, the errors in the $X$ (north), $Y$ (east) and $Z$ components remain constant at $140 \mathrm{nT}$.

To update the observation made in 2005 to the time of the 2007 observations, the annual change at the site was calculated using the 10th generation IGRF (Macmillan and Maus, 2005). The annual changes for 2005 and 2006 were also calculated for the sites of RES and THL observatories. These were compared to the observed annual change. The differences constitute the model error at those locations. These were used to estimate corrections to the IGRF annual change values for the observation site. For example, the SV at Resolute obtained from the IGRF is $93 \mathrm{nT}$ per year in $X$. The observed SV, based on annual mean values, is $78 \mathrm{nT}$ per year, a difference of $15 \mathrm{nT}$. The observed SV at Thule was also $15 \mathrm{nT}$ less than the IGRF value. We therefore assumed a correction of $15 \mathrm{nT}$ per year which we applied to the SV obtained from the IGRF for observation site $05-01$ for the time interval 2005.4 to 2007.3. The other components were updated in a similar manner. 


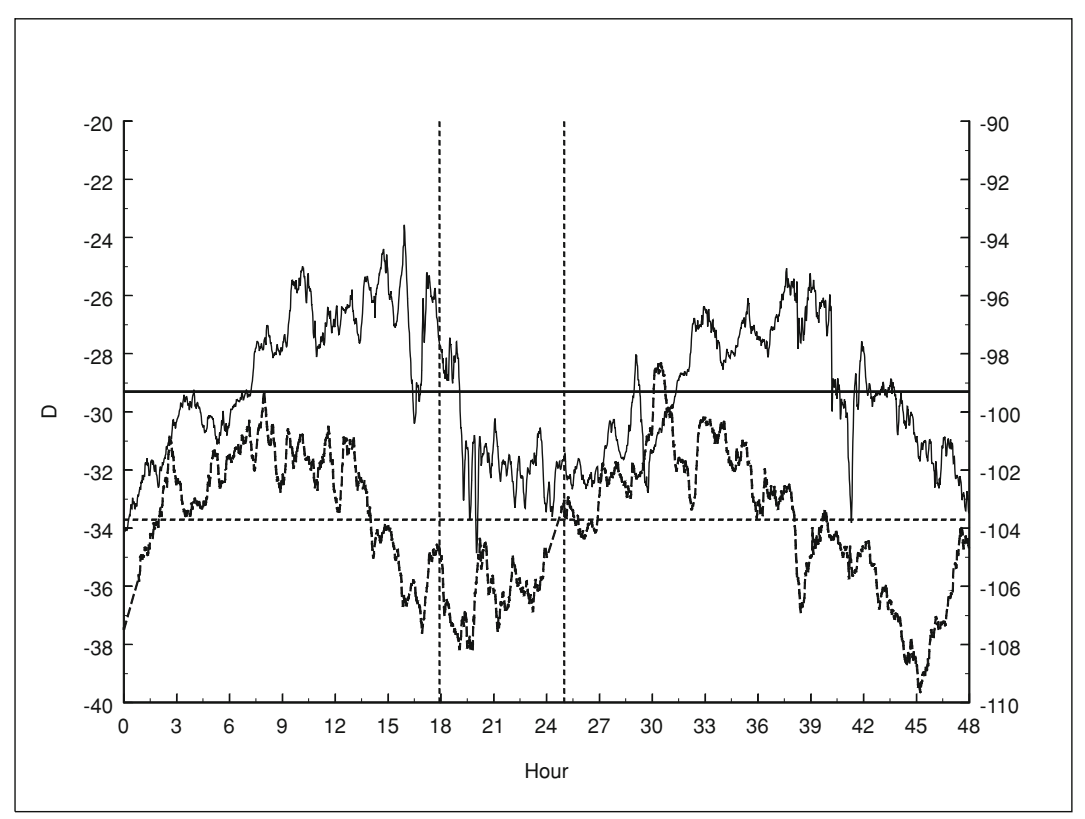

Fig. 2. Magnetic field variations recorded on April 29 and April 30, 2007 at Resolute Bay magnetic observatory (upper) and Eureka magnetometer station (lower). The horizontal lines denote the average declination at Resolute (solid) and Eureka (dashed) during the two day period. The dashed vertical lines indicate the time interval of the survey.

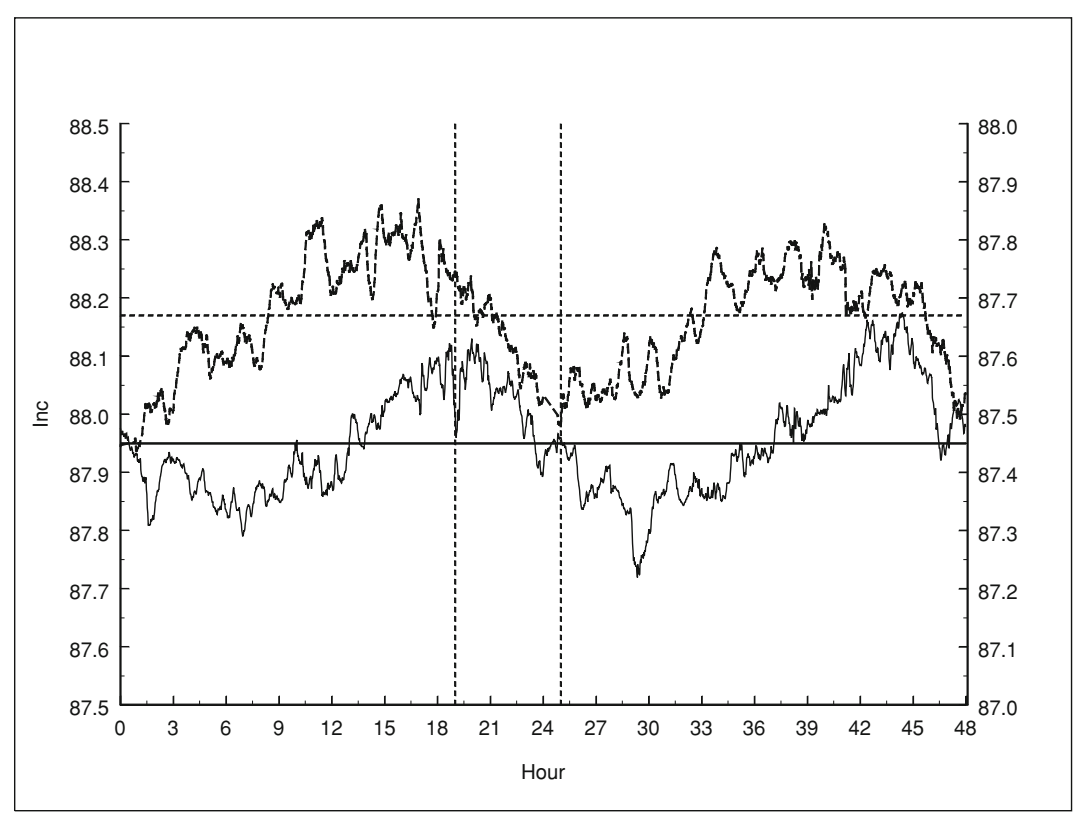

Fig. 3. Same as Fig. 2 but for inclination. Note that Eureka is the upper trace.

\section{Determination of the North Magnetic Pole Po- sition}

There are several possible ways by which a NMP position can be calculated from a sparse set of observations. We examine four methods of differing degrees of complexity and validity; these are described in the following sections. The position of the NMP that we adopt is the average of the positions computed using each of these methods (Table 3). The positional uncertainty is calculated by multiplying the horizontal gradient by the observational or model error. The gradient in inclination is extremely heterogeneous, ranging from approximately $300 \mathrm{~km}$ per degree in the south-north direction to about $200 \mathrm{~km}$ per degree in the east-west direc- tion. Both the model derived by a spherical cap harmonic analysis (SCHA), and the polynomial model fit the observations to approximately $250 \mathrm{nT}$ which equates to approximately $0.25^{\circ}$ in inclination. The positional uncertainty is therefore about $40 \mathrm{~km}$ north-south, or approximately $0.4 \mathrm{de}$ grees of latitude. In the east-west direction the longitudinal error is approximately 1.2 degrees.

\subsection{VGP method}

Assuming a dipole field, inclination and declination can be used to calculate distance and bearing to the geomagnetic pole, which would be the same as the NMP. This is identical to the calculation of virtual geomagnetic poles in paleomagnetism (see Merrill et al., 1998). Pole positions calculated 
Table 3.

\begin{tabular}{lcc}
\hline \multicolumn{1}{c}{ Method } & Latitude $(\mathrm{N})$ & Longitude $(\mathrm{W})$ \\
\hline VGP method & 83.96 & 119.80 \\
Simple polynomial & 83.99 & 122.80 \\
Spherical cap harmonics & 83.89 & 120.22 \\
Grid shift & 83.96 & 120.05 \\
Average & 83.95 & 120.72 \\
\hline
\end{tabular}

from each of the five observations should be tightly clustered. However, this is far from the case. The five poles have an angular dispersion of greater than $1^{\circ}$. The underlying assumption - that the Earth's magnetic field is essentially dipolar-is invalid in the north polar region. As a result, the horizontal gradient of the field is not homogenous, nor does the magnetic declination give the azimuth of the NMP. Simulations carried out using the IGRF indicate that pole positions calculated using five observations will differ from the true position by distances that range from $18 \mathrm{~km}$ to $185 \mathrm{~km}$, depending on the locations of the five observations. Therefore, the close agreement between the pole position obtained using this method with the pole positions obtained from the other three methods may simply be fortuitous.

\subsection{Simple polynomials}

A simple approach to determining the NMP position is to fit a low order polynomial in latitude $(\theta)$ and longitude $(\lambda)$ to the $X$ and $Y$ components:

$$
X=c 1 * \theta+c 2 * \lambda+c 3 * \theta * \lambda+c 4
$$

The polynomials can then be used to compute values of $X$ and $Y$ on a dense grid over a region that included the likely location of the NMP. The point at which $X=Y=0$ is the NMP. Note that because $X$ and $Y$ are fitted separately they are not necessarily mutually consistent and do not form a solution to Laplace's equation $\nabla^{2} V=0$, where $V$ is the scalar potential of the magnetic field.

\subsection{Spherical cap harmonic analysis}

Spherical cap harmonic analysis (Haines, 1985) has been used several times to compute models based on localized NMP surveys that can then be used to determine the NMP position (Newitt and Barton, 1996; Newitt et al., 2002). SCHA is superior, at least in theory, to polynomial analysis because all magnetic field components enter the solution. Thus, no information is wasted, and the model is a solution to Laplace's equation. However, when a spherical cap harmonic analysis was carried out on the five observations, the solution was unstable. It was necessary to add synthetic data (calculated from the IGRF) near the periphery of the spherical cap. The resultant model was used to find the point at which $X=Y=0$ as with the simple polynomials.

The use of synthetic data, although necessary to obtain a stable solution, does introduce another source of error since we are essentially introducing an offset around the five observation points that the model tries to reproduce, thereby giving a set of SCH coefficients that differ from the set that would be obtained if the synthetic data could be replaced by real data. Simulations indicate that the corresponding error in the pole position will be small, approximately $10 \mathrm{~km}$.

\subsection{Best fit to grid data}

A simple approach that takes into account the non-dipole nature of the field is to compare the observed values at the 5 sites to the values given by a reference field model. We used the CHAOS model (Olsen et al., 2006). The model was used to calculate the $X$ and $Y$ component values for the five sites. The differences between the observed and model values were computed, from which a goodness of fit parameter was derived. This was simply the sum of the squares of the differences for the $X$ and $Y$ components. We then incrementally rotated the isolines, shifted the latitude and longitude, and recomputed the goodness of fit parameter. This procedure was repeated until we found a point where the fit parameter was a minimum. The latitudinal and longitudinal displacements were noted and applied to the model NMP position. The displaced NMP position can be considered the observed position.

\subsection{The effect of crustal anomalies}

The projected location of the NMP in 2007 places it near or possibly on the Alpha Ridge, a prominent structure in the Arctic Ocean featuring some large magnetic anomalies. These could possibly contaminate the observations made during the NMP survey. We therefore decided to investigate this possibility by examining the $100 \mathrm{~m}$ grid of total intensity data based on aeromagnetic data, available from the Geological Survey of Canada. The grid is based on aeromagnetic surveys flown at an altitude of approximately $130 \mathrm{~m}$. The data are relative total intensity data $(d F)$; that is, a reference field of unknown origin (but presumably a version of the IGRF) has been subtracted. Figure 4 shows a contour plot of $d F$ in the region containing the four sites visited in 2007. The values of $d F$ for the sites, scaled from the contour plot, are given in Table 4. We compared these to difference values obtained by subtracting the most recent IGRF model from the observed values $\left(d F_{\mathrm{i}}\right)$. We also computed difference values using the CHAOS model to degree and order $14\left(d F_{\mathrm{c}}\right)$. In theory, these should be almost identical to $d F$, but differences in the accuracy of the dif-

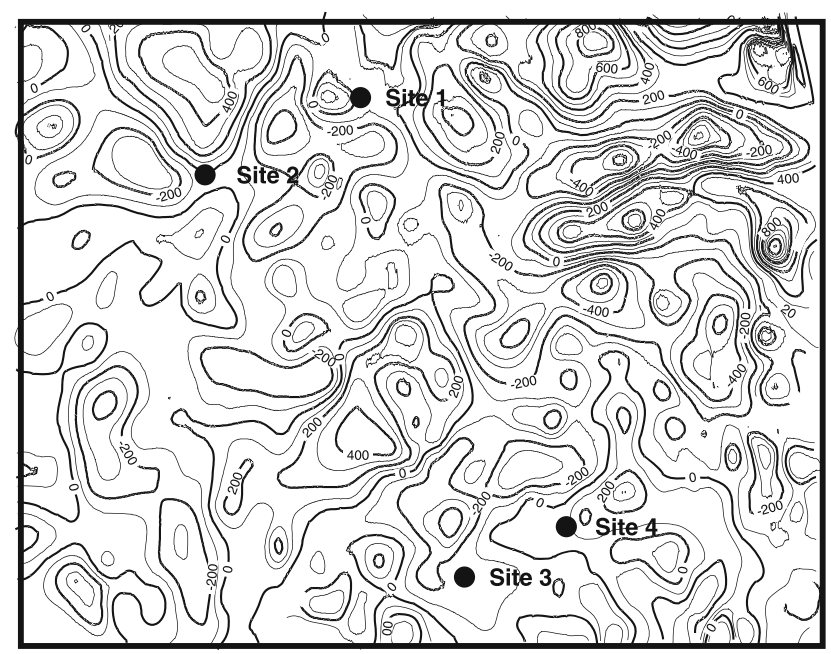

Fig. 4. Contour map of relative total intensity in the vicinity of the North Magnetic Pole. 
Table 4.

\begin{tabular}{crrrcc}
\hline Site & $d F(\mathrm{nT})$ & $d F_{\mathrm{i}}(\mathrm{nT})$ & $d F_{\mathrm{c}}(\mathrm{nT})$ & $I_{\mathrm{obs}}-I_{\text {igrf }}\left({ }^{\circ}\right)$ & $I_{\text {obs }}-I_{\text {chaos }}\left({ }^{\circ}\right)$ \\
\hline $07-01$ & -60 & -140 & -149 & .050 & .068 \\
$07-02$ & 20 & 46 & 55 & -0.361 & -.355 \\
$07-03$ & -175 & -131 & -101 & .095 & .123 \\
$07-04$ & 75 & 142 & 160 & -0.042 & -.008 \\
$05-01$ & 50 & 61 & 98 & 0.575 & 0.600 \\
\hline
\end{tabular}

Table 5.

\begin{tabular}{c|cc|ccc|ccc}
\hline Year & \multicolumn{2}{|c|}{ Observed } & \multicolumn{3}{c|}{ IGRF } & \multicolumn{3}{c}{ Chaos } \\
\hline & Lat & Long & Lat & Long & Dist $(\mathrm{km})$ & Lat & Long & Dist $(\mathrm{km})$ \\
\hline 2007.3 & 83.95 & 120.72 & 84.25 & 124.38 & 50.2 & 83.91 & 123.80 & 27.2 \\
2001.0 & 81.30 & 110.80 & 81.44 & 111.02 & 20.1 & 81.34 & 111.24 & 8.6 \\
1994.0 & 78.30 & 104.0 & 78.85 & 104.94 & 64.6 & & & \\
1983.9 & 77.0 & 102.3 & 77.29 & 102.40 & 32.3 & & & \\
1973.5 & 76.0 & 100.6 & 76.07 & 100.74 & 8.6 & & & \\
1962.5 & 75.1 & 100.8 & 75.46 & 101.17 & 41.4 & & & \\
1948.0 & 73.9 & 100.9 & 74.35 & 100.60 & 50.9 & & & \\
1904.5 & 70.5 & 96.6 & 70.64 & 96.45 & 16.6 & & & \\
1831.4 & 70.1 & 96.9 & & & & & & \\
\hline
\end{tabular}

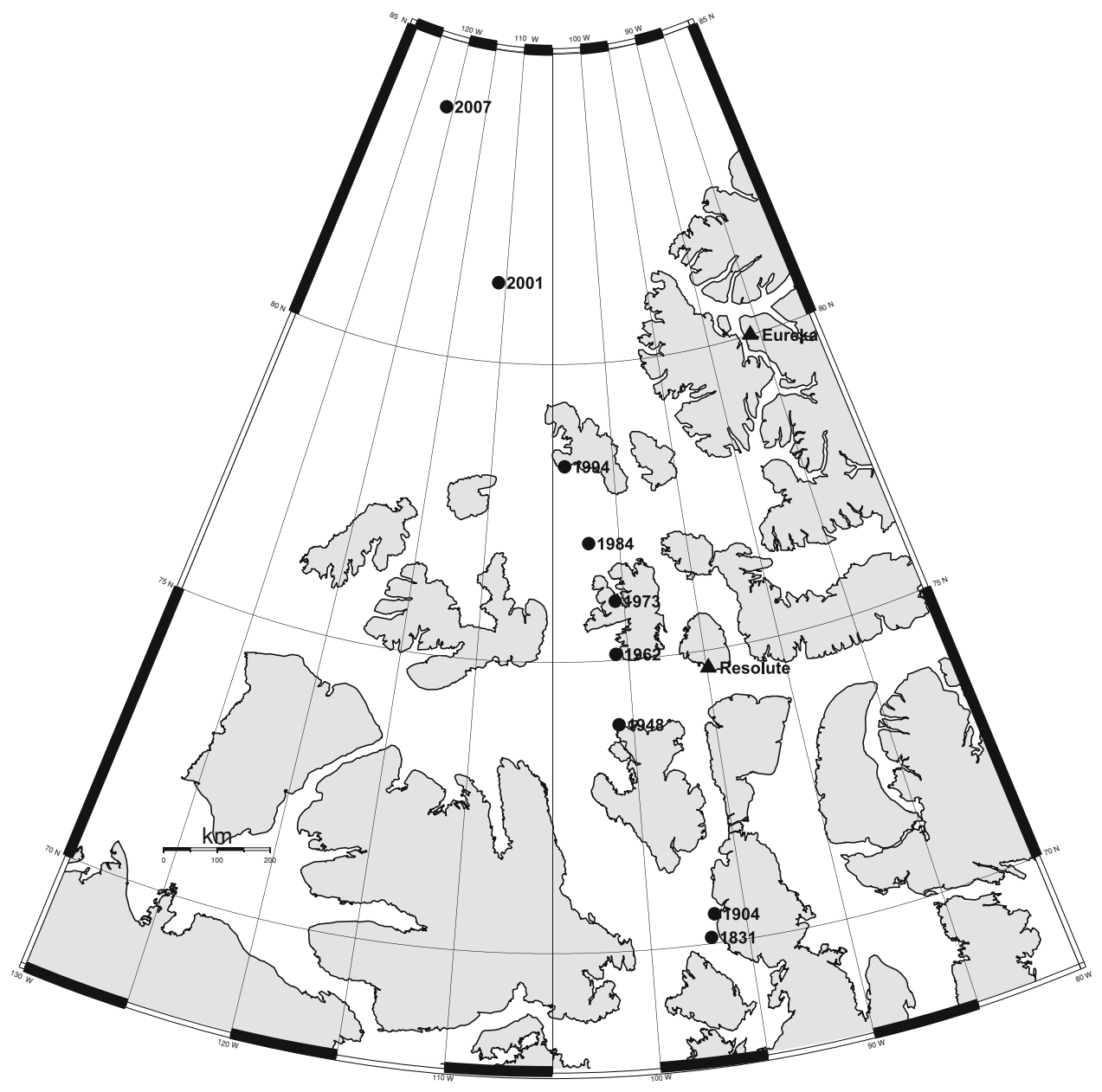

Fig. 5. Observed positions of the North Magnetic Pole.

ferent models and the presence of errors due to magnetic disturbances of external origin will almost certainly lead to differences between $d F, d F_{\mathrm{i}}$ and $d F_{\mathrm{c}}$.

Because inclination is the most important component for the determination of the NMP position, we computed the differences in inclination between the observed values and the IGRF. These are also given in Table 4.

Figure 4 and Table 4 show that $d F$ ranges from -175 (at 
Table 6.

\begin{tabular}{ccccccc}
\hline Time Span & $\begin{array}{c}\text { Speed } \\
\text { (Observed) } \\
\mathrm{km} / \mathrm{yr}\end{array}$ & $\begin{array}{c}\text { Speed } \\
\text { (IGRF) } \\
\mathrm{km} / \mathrm{yr}\end{array}$ & $\begin{array}{c}\text { Speed } \\
\text { (CHAOS) } \\
\mathrm{km} / \mathrm{yr}\end{array}$ & Time Span & $\begin{array}{c}\text { Acceleration } \\
\text { (Observed) } \\
\mathrm{km} / \mathrm{yr}^{2}\end{array}$ & $\begin{array}{c}\text { Acceleration } \\
\text { (IGRF) } \\
\mathrm{km}^{2} \mathrm{yr}^{2}\end{array}$ \\
\hline 1904.5 to 1948.0 & 9.3 & 10.0 & & 1926.25 to 1955.25 & 0.0 & -0.05 \\
1948.0 to 1962.5 & 9.2 & 8.6 & & 1955.25 to 1968.0 & 0.0 & -0.18 \\
1962.5 to 1973.5 & 9.1 & 6.3 & & $1968 / 0$ to 1978.7 & 0.2 & 0.69 \\
1973.5 to 1983.9 & 11.3 & 13.6 & & 1978.7 to 1989.0 & 0.35 & 0.40 \\
1983.9 to 1994.0 & 14.9 & 18.1 & & 1989.0 to 1997.0 & 4.66 & 3.15 \\
1994.0 to 2001.0 & 51.5 & 43.8 & & 1997.0 to 2004.15 & 0.1 & 2.07 \\
2001.0 to 2007.3 & 52.0 & 58.1 & 53.3 & & & \\
\hline
\end{tabular}

Site 07-03) to $75 \mathrm{nT}$. There is some crustal contamination but none of the sites is highly anomalous. Table 4 shows that Site 05-01, not Site 07-03, has the largest inclination anomaly.

\section{The Movement of the North Magnetic Pole}

Table 5 lists all observed positions of the NMP along with positions calculated from the IGRF and CHAOS geomagnetic field models. The observed positions are plotted in Fig. 5. IGRF models have been produced for each five year epoch since 1900 (Macmillan and Maus, 2005). Field values (and derived pole positions) for years other than model epochs are obtained by linear interpolation between models. There are, however, linear secular variation terms to allow extrapolation beyond the final epoch model of the model (2005). For the observed position at 2007.3 we have used the average position obtained from the four methods listed in Table 3. The column labelled "distance" refers to the distance between the observed and model positions. For the most part, the differences are easily explainable in terms of model error and observational error, in particular inadequate reduction to the quiet level.

The average rate of motion of the NMP between observations is given in Table 6. Rates of motion were calculated using the IGRF positions as well. The table also shows the observed and model acceleration of the NMP. The motion of the NMP was relatively constant up to 1994, at which time an intense acceleration occurred. However, whereas direct observation indicates that the NMP was no longer accelerating between 1997 and 2004.15, the IGRF shows a continued acceleration over the same time period. Based on the latest velocity and acceleration derived from the observed values, the NMP will have travelled $660 \mathrm{~km}$ by 2020 and will be located at $87.8^{\circ} \mathrm{N}, 172.5^{\circ} \mathrm{E}$. Using values derived from the IGRF places the pole at $86.3^{\circ} \mathrm{N}, 119^{\circ} \mathrm{E}$, a distance of $905 \mathrm{~km}$ from its current location. However, such an extrapolation is essentially meaningless since the differences between the observed and modelled positions that give rise to the large difference between the observed acceleration and the IGRF model acceleration are not statistically meaningful.

\section{Conclusions}

The survey carried out in April, 2007, gives a position for the North Magnetic Pole that is in close agreement with the position obtained from both the IGRF and CHAOS mod- els. However, the observations and models give conflicting predictions of the NMP's motion in the coming decades. A year-by-year analysis of the NMP position derived from the CHAOS model suggests that the NMP started to decelerate between 2001 and 2007 (Olsen and Mandea, 2007). The time interval between observations is too large to allow us to confirm or contradict this suggestion, with any degree of certainty, but it appears likely that the NMP is no longer accelerating. The IGRF, on the other hand, indicates continued acceleration, albeit at a reduced rate.

The logistic difficulties associated with a survey of the NMP have increased to the point that such surveys may no longer be practical. Fortunately, models based on high quality satellite data give NMP locations with an accuracy that is likely superior to those obtained by a ground survey. However, ground surveys have provided an excellent form of public outreach over the years, and this will be missed.

Acknowledgments. This expedition was largely privately funded by the Poly-arctic project and the following sponsors: TAITBOUT Group, ABBOTT Laboratory, AUDIENS Group, Kenn Borek Air, NOVALIS Group, Grand Nord-Grand Large Ltd, French Research Ministry. We also wish to acknowledge the support of M. Angatookaluk and M. Orgeval and the staff of the Eureka Weather Station. Magnetometer data for Resolute and Eureka, and the aeromagnetic grid data were supplied by the Geological Survey of Canada. This is IPGP contribution number 2449.

\section{References}

Campbell, W. H., Comments on "Survey Tracks Current Position of South Magnetic Pole" and "Recent Acceleration of North Magnetic Pole Linked to Magnetic Jerks", Eos Trans., AGU, 84, 41, 2003.

Fraser-Smith, A., Centered and eccentric geomagnetic dipoles and their poles, 1600-1985, Rev. Geophys., 25, 1-16, 1987.

Haines, G. V., Spherical cap harmonic analysis, J. Geophys. Res., 90, 2583-2591, 1985.

Lowes, F. J., The geomagnetic eccentric dipole: Facts and fallacies, Geophys. J. Int., 118, 671-679, 1994.

Macmillan, S. and S. Maus, International geomagnetic reference field - the tenth generation, Earth Planets Space, 57, 1135-1140, 2005.

Mandea, M. and M. Dormy, Asymmetric behaviour of the magnetic dip poles, Earth Planets Space, 55, 153-157, 2003.

Merrill, R. T., M. W. McElhinny, and P. L. McFadden, The magnetic field of the Earth, paleomagnetism, the core and the deep mantle, International Geophysics Series Volume 63, Academic Press, 531 pp., 1998.

Newitt, L. R. and C. E. Barton, The position of the North Magnetic Pole in 1994, J. Geomag. Geoelectr., 48, 221-232, 1996.

Newitt, L. R., C. E. Barton, and J. Bitterly, IAGA guide for magnetic repeat station surveys, International Association of Geomagnetism and Aeronomy special publications, 1996.

Newitt, L. R., M. Mandea, L. A. McKee, and J.-J. Orgeval, Recent acceleration of the North Magnetic Pole linked to magnetic jerks, Eos, Trans. 
$A G U, \mathbf{8 3}, 381,2002$

Olsen, N., H. Lühr, T. Sabaka, M. Mandea, M. Rother, L. Toffner-Clausen, and S. Choi. CHAOS - a model of the Earth's magnetic field derived from CHAMP, Ørsted, and SAC-C magnetic satellite data, Geophys. J. Int., 166, 67-75, 2006.

Olsen, N. and M. Mandea, Will the magnetic north pole wind up in Sibeia, Eos, Trans. AGU, 88, 293, 2007.
Ross, J. C., On the position of the North Magnetic Pole, Phil. Trans. Roy. Soc. London, 124, 46-51, 1834.

L. R. Newitt (e-mail: lnewitt@sympatico.ca), A. Chulliat, and J.-J. Orgeval 